\title{
A Hipersensibilidade Pulmonar E O Sars-Cov-2: Uma Revisão Da Literatura
}

\section{Lung Hypersensitivity and Sars-Cov-2: A Literature Review}

\section{Nathália Perini Zamprogno}

Acadêmica de Medicina na EMESCAM - Escola Superior de Ciências da Santa Casa de Misericórdia de Vitória

Endereço institucional: Avenida Nossa Senhora da Penha, 2190, Santa Luiza, Vitória, ES

E-mail: nathalia.perini@hotmail.com

\section{Maria Clara Biccas Braga}

Acadêmica de Medicina na EMESCAM - Escola Superior de Ciências da Santa Casa de Misericórdia de Vitória

Endereço institucional: Avenida Nossa Senhora da Penha, 2190, Santa Luiza, Vitória, ES

E-mail: mariaclarabbraga@outlook.com

\section{Shayra Tofano Monteiro}

Acadêmica de Medicina na EMESCAM - Escola Superior de Ciências da Santa Casa de Misericórdia de Vitória

Endereço institucional: Avenida Nossa Senhora da Penha, 2190, Santa Luiza, Vitória, ES

E-mail: shayratofanomonteiro@gmail.com

\section{Manuela Marchesi}

Acadêmica de Medicina na EMESCAM - Escola Superior de Ciências da Santa Casa de Misericórdia de Vitória

Endereço institucional: Avenida Nossa Senhora da Penha, 2190, Santa Luiza, Vitória, ES

E-mail:manubmarchesi@gmail.com

\section{Luísa Pirola Santos}

Acadêmica de Medicina na EMESCAM - Escola Superior de Ciências da Santa Casa de Misericórdia de Vitória

Endereço institucional: Avenida Nossa Senhora da Penha, 2190, Santa Luiza, Vitória, ES

E-mail: luisapirolasantos@gmail.com

\section{Marcos Guilherme Bedim Trancoso}

Acadêmica de Medicina na EMESCAM - Escola Superior de Ciências da Santa Casa de Misericórdia de Vitória 
Endereço institucional: Avenida Nossa Senhora da Penha, 2190, Santa Luiza, Vitória, ES

E-mail: marcosbedimtrancoso@hotmail.com

\section{Isadora Liparizi}

Acadêmica de Medicina na EMESCAM - Escola Superior de Ciências da Santa Casa de Misericórdia de Vitória

Endereço institucional: Avenida Nossa Senhora da Penha, 2190, Santa Luiza, Vitória, ES

E-mail: isadoradeoliveiraliparizi@gmail.com

\section{Joana Degasperi Diniz}

Acadêmica de Medicina na EMESCAM - Escola Superior de Ciências da Santa Casa de Misericórdia de Vitória

Endereço institucional: Avenida Nossa Senhora da Penha, 2190, Santa Luiza, Vitória, ES

E-mail: joanadegasperi.diniz@gmail.com

\section{Amanda Vivacqua Figueiredo}

Acadêmica de Medicina na EMESCAM - Escola Superior de Ciências da Santa Casa de Misericórdia de Vitória

Endereço institucional: Avenida Nossa Senhora da Penha, 2190, Santa Luiza, Vitória, ES.

E-mail: amandavivacquafigueiredo@gmail.com

\section{Luciene Lage da Motta}

Graduação em medicina pela Escola Superior de Ciências da Santa Casa de Misericórdia de Vitória(1997) e residencia-medicapela Santa Casa de Misericórdia de São Paulo(2001)

Endereço institucional: Avenida Nossa Senhora da Penha, 2190, Santa Luiza, Vitória, ES.

E-mail: lucine.motta@emescam.br

\section{RESUMO}

Introdução: Desde o final de 2019 o mundo enfrenta o aparecimento de um novo coronavírus, o SARS-COV-2, responsável pelo surgimento da pandemia de COVID-19. Estudos indicam que a hipersensibilidade pulmonar (HP) é responsável pela falência respiratória aguda/hipóxica provocada pelos casos graves de COVID-19. A evolução da HP depende de vários fatores, como tamanho da partícula, solubilidade, tempo de exposição, alterações no sistema imune do hospedeiro, genética e questões ambientais. A apresentação clínica é complexa, na qual evidenciam-se sintomas gripais como febre, tosse, aperto no peito, dispneia e a progressividade da doença pode trazer consequências crônicas, como, por exemplo, fibrose pulmonar. Objetivo: Investigar a correlação existente entre a hipersensibilidade pulmonar e a patogênese provocada pelo vírus SARSCOV-2. Metodologia: Revisão bibliográfica realizada na bases de dados PubMed/MEDLINE, em setembro de 2021, por meio do cruzamento dos descritores, cadastradas no DeCS, "COVID-19", "SARS-COV-2" e "Hypersensitivity Pneumonitis".Utilizou-se como critérios de inclusão artigos publicados nos últimos cinco anos, nos idiomas inglês, português ou 
espanhol.Foram excluídos aqueles cujo título ou resumo eram incoerentes com a linha de interesse da atual revisão. Com base nisso, encontraram-se 10 artigos, dos quais 3 foram selecionados, além de outro artigo, julgado importante para a discussão do tema. Resultados e discussões: No final de 2019 surgiu a pandemia de COVID-19, responsável por inúmeros óbitos e por manifestações clínicas de febre, tosse, dispneia, fadiga, dentre outros. Ainda, vale ressaltar a existência da chamada pneumonite de hipersensibilidade (HP), um processo inflamatório em resposta a um antígeno, que causa sintomas similares aos citados anteriormente, tais como febre, tosse, dispnéia, podendo acarretar até mesmo em doenças crônicas. Nesse contexto, é importante salientar que a progressão sintomatológica do COVID e HP são semelhantes, partindo de sintomas clássicos gripais até um quadro de severa ativação da resposta imune, sendo imperativo a diferenciação das patologias citadas acima. No entanto, o achado radiológico e patológico típico em ambas as doenças são semelhantes, com opacidades em vidro fosco, consolidativas ou nodulares, com o tipo de distribuição sendo periférica, bilateral e multilobar, podendo incluir ainda padrão de pavimentação em mosaico (com ou sem consolidação ou linhas intralobulares visíveis), espessamento do septo interlobular, bronquiectasia ou envolvimento subpleural. Considerações finais: Existe uma correlação do achado radiológico e patológico entre a HP e a pneumonia do COVID-19:opacidades em vidro fosco, consolidativas ou nodulares, com o tipo de distribuição sendo periférico, bilateral e multilobar. O contexto pandêmico influência na suspeita diagnóstica, por isso, é necessário exames específicos de confirmação para que 0 tratamento seja adequado. Portanto,cautela, atenção, conhecimento e individualidade irão determinar a regressão da doença.

Palavras chave: COVID-19, Hipersensibilidade Pulmonar, Inflamação Pulmonar.

\section{ABSTRACT}

Introduction: Since late 2019, the world has faced the emergence of a new coronavirus, SARS-COV-2, responsible for the emergence of the COVID-19 pandemic. Studies indicate that pulmonary hypersensitivity $(\mathrm{PH})$ is responsible for the acute respiratory/hypoxic failure caused by severe cases of COVID-19. The evolution of $\mathrm{PH}$ depends on several factors, such as particle size, solubility, exposure time, changes in the host immune system, genetics, and environmental issues. The clinical presentation is complex, with flu-like symptoms such as fever, cough, chest tightness, and dyspnea, and the progression of the disease can lead to chronic consequences, such as pulmonary fibrosis. Objective: To investigate the correlation between pulmonary hypersensitivity and the pathogenesis caused by the SARS-COV-2 virus. Methodology: Bibliographic review conducted in the PubMed/MEDLINE database in September 2021 by crossing the descriptors, registered in DeCS, "COVID-19", "SARS-COV-2" and "Hypersensitivity Pneumonitis", using as inclusion criteria articles published in the last five years in English, Portuguese or Spanish. Based on this, 10 articles were found, of which 3 were selected, in addition to another article, judged important for the discussion of the theme. Results and discussions: In late 2019 the pandemic of COVID-19 appeared, responsible for numerous deaths and for clinical manifestations of fever, cough, dyspnea, fatigue, among others. Also, it is worth mentioning the existence of the so-called hypersensitivity pneumonitis (HP), an inflammatory process in 
response to an antigen, which causes symptoms similar to those mentioned above, such as fever, cough, dyspnea, and may even lead to chronic diseases. In this context, it is important to note that the symptomatological progression of COVID and HP are similar, from classic flu-like symptoms to a severe activation of the immune response, making it imperative to differentiate the aforementioned pathologies. However, the typical radiological and pathological findings in both diseases are similar, with ground-glass opacities, consolidative or nodular, with the type of distribution being peripheral, bilateral, and multilobar, and may also include a mosaic pattern of paving (with or without consolidation or visible intralobular lines), interlobular septal thickening, bronchiectasis, or subpleural involvement. Final considerations: There is a correlation of radiological and pathological finding between PH and pneumonia of COVID-19: ground-glass opacities, consolidative or nodular, with the type of distribution being peripheral, bilateral and multilobar. The pandemic context influences diagnostic suspicion, so specific confirmatory tests are required for appropriate treatment. Therefore, caution, attention, knowledge and individuality will determine the regression of the disease.

Key words: COVID-19, Pulmonary hypersensitivity, Pulmonary inflammation.

\section{INTRODUÇÃO}

Desde o final de 2019 o mundo enfrenta o aparecimento de um novo coronavírus, o SARS-COV-2, responsável pelo surgimento da pandemia de COVID-19. Estudos indicam que a hipersensibilidade pulmonar (HP) é responsável pela falência respiratória aguda/hipóxica provocada pelos casos graves de COVID-19. A evolução da HP depende de vários fatores, como tamanho da partícula, solubilidade, tempo de exposição, alterações no sistema imune do hospedeiro, genética e questões ambientais. A apresentação clínica é complexa, na qual evidenciam-se sintomas gripais como febre, tosse, aperto no peito, dispneia e a progressividade da doença pode trazer consequências crônicas, como, por exemplo, fibrose pulmonar.

\section{OBJETIVO}

Investigar a correlação existente entre a hipersensibilidade pulmonar e a patogênese provocada pelo vírus SARS-COV-2.

\section{MÉTODO}

Trata-se de uma revisão bibliográfica com abordagem expositiva e analítica acerca da associação entre o SARS-COV-2 e a hipersensibilidade pulmonar. Para 
tal, foram feitas consultas à PubMed (US National Library of Medicine) em setembro de 2021 por meio do cruzamento dos descritores "COVID-19", "SARSCOV-2" e "Hypersensitivity Pneumonitis". Desse modo, pesquisou-se por "("SARS-COV-2" OR "COVID-19") AND "Hypersensitivity Pneumonitis"' para encontrar artigos de interesse. Foram considerados somente artigos disponíveis em texto completo e que foram publicados nos últimos cinco anos, nas línguas inglesa ou portuguesa, sendo encontrado um " $n$ " de 10 artigos. Os critérios de seleção utilizados foram exclusão por título e por resumo, e, após a aplicação destes, selecionou-se 3 artigos. Acrescentou-se também 0 estudo "Hypersensitivity in the lungs is responsible for acute respiratory failure in COVID19 patients: Case series of patients who received high-dose/short-term methylprednisolone", não encontrado por meio das estratégias de busca apresentadas, a fim de embasar essa revisão da literatura.

Figura 1: Fluxograma de seleção de artigos na revisão sobre a relação entre o vírus SARS-CoV-2 e a Hipersensibilidade Pulmonar.

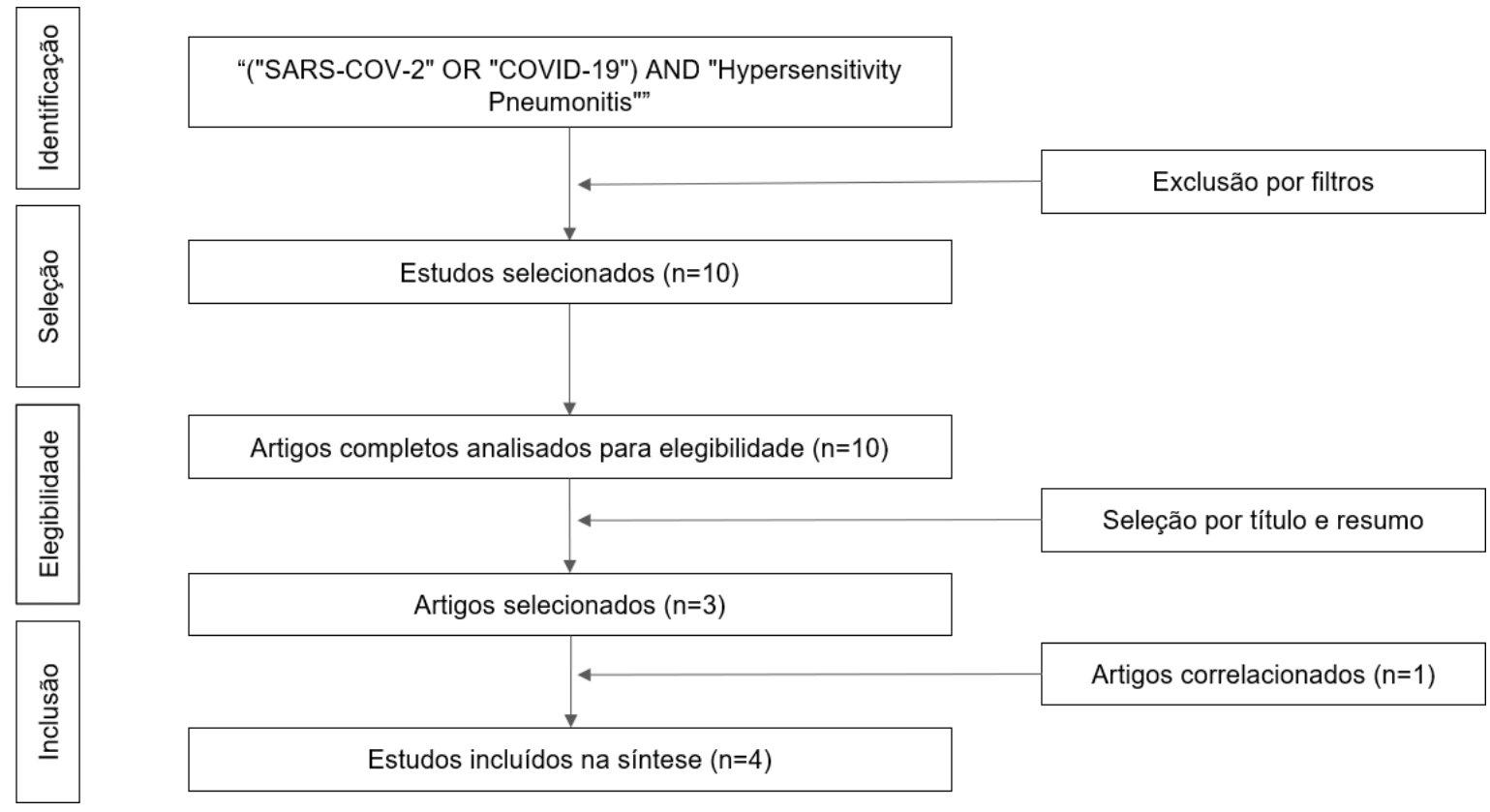

\section{DISCUSSÃO}

Desde o final de 2019 o mundo enfrenta o aparecimento de um novo coronavírus, o SARS-COV-2, responsável pelo surgimento da pandemia de COVID-19. A infecção por esse vírus possui como principais manifestações febre, tosse, dispneia, mialgia, fadiga, sintomas respiratórios superiores e sintomas 
gastrointestinais com intensidade variável. Estudos indicam que a hipersensibilidade pulmonar é responsável pela falência respiratória aguda/hipóxica provocada pelos casos graves de COVID-19. ${ }^{4}$

Tratando-se de hipersensibilidade pulmonar, a pneumonite de hipersensibilidade, ou Hypersensitivity Pneumonitis (HP), também conhecida como alveolite alérgica extrínseca, é uma combinação de hipersensibilidade tipo III e IV, com uma presença aumentada de complexos imunológicos e atividade em cascata do sistema complemento. ${ }^{3}$ A HP se configura como um processo inflamatório em resposta a um antígeno, o qual pode ser infeccioso (fungos, bactérias, vírus) ou por inalação frequente de partículas prejudiciais, como poeira e isocianato, que é utilizado na produção de polímeros, em um indivíduo sensibilizado. A evolução da HP depende de vários fatores, como tamanho da partícula, solubilidade, tempo de exposição, alterações no sistema imune (inato e adaptativo) do hospedeiro, genética e questões ambientais. A apresentação clínica é complexa, na qual evidenciam-se sintomas gripais como febre, tosse, aperto no peito, dispneia e a progressividade da doença pode trazer consequências crônicas, como, por exemplo, fibrose pulmonar. ${ }^{3}$

A alveolite alérgica extrínseca apresenta-se em três variantes de acordo com sua fisiopatologia: aguda, subaguda e crônica. $O$ tipo agudo se manifesta especialmente em idosos, uma vez que há declínio da imunidade adaptativa em pessoas de idade mais avançada, a imunidade inata não é eficaz e há ativação acelerada do sistema complemento, ocasionando uma grave pneumonite de progressão rápida. Já um adulto saudável, com imunidade adaptativa normal, tende a desenvolver a HP do tipo subaguda, já que apresenta melhor controle da reação inflamatória, por meio da regulação imunológica, enquanto a população pediátrica apresenta sintomas mínimos ou ausentes em decorrência da ativação do sistema imune inato. ${ }^{1}$

Dito isso, sabe-se que em casos severos de infecção por SARS-CoV-2 há uma progressão da sintomatologia, partindo de sintomas clássicos como tosse e dispneia para um quadro de severa ativação da resposta imune, sendo observadas na Fase 3 da progressão do COVID-19 (8ำ - 9 dia), infiltração inflamatória intersticial, acúmulo de fluidos e fibrose progressiva nos pulmões, gerando comprometimento da troca de gases (Síndrome do Desconforto 
Respiratório Agudo), ao qual podem se associar a insuficiência ventricular direita e o risco aumentado de tromboembolismo pulmonar. Essas características são compatíveis com aquelas observadas na progressão da HP, assim, sugere-se que as fases de evolução da COVID-19 possam mimetizar essa patologia de desregulação da imunidade. Por conseguinte, propõe-se que a doença causada pelo coronavírus SARS-CoV-2 seja capaz de desencadear uma reação imune semelhante à pneumonite de hipersensibilidade. ${ }^{3}$

\subsection{IMUNOPATOGÊNESE}

A hipersensibilidade crônica e o COVID-19, na sua forma grave, podem progredir para a hiperinflamação dos pulmões. Níveis elevados da imunoglobulina $\operatorname{lgE}$ específica anti-SARS- CoV-2 indicam hipersensibilidade pulmonar provocada pelo vírus. Nesse contexto, complexos imunes com atividade de células T começam a ser desenvolvidos e a cascata do sistema complemento se estabelece. A principal característica é a presença de atividade eosinofílica e resposta Th2. Os eosinófilos, por meio de suas ribonucleases via receptor TLR7, degradam o genoma do RNA viral presente no trato respiratório.

O processo de sinalização celular inclui a expressão de interleucinas IL-6 (caracteriza casos graves de COVID-19) e TNF, estimulados quase 2 vezes mais pelos receptores TLR7 do que quando a infecção é por outro vírus. Além disso, ocorre a liberação de IL-4 e IL-13 por mastócitos, que estimula a recrutação de eosinófilos da medula óssea para o pulmão e de IL-5, uma citocina importante e essencial para a eliminação do vírus. $O$ fato dos eosinófilos serem recrutados para os pulmões diminui sua quantidade no sangue, indicando a presença de eosinopenia. Nesse aspecto laboratorial, encontra-se também uma transição do número de neutrófilos para linfócitos nos pacientes graves que permaneceram no tratamento intensivo. ${ }^{3}$

\subsection{MÉTODOS DIAGNÓSTICOS}

Exames importantes para o diagnóstico e monitoramento da HP são as radiografias e tomografias computadorizadas (TC). Inicialmente, nas radiografias, a doença pode se manifestar por meio de numerosas pequenas opacidades nodulares mal definidas em ambos os pulmões, poupando ápices e bases, além 
de edema pulmonar de opacidade em padrão vidro fosco irregular ou difuso. Entretanto, a sensibilidade da radiografia de tórax para a detecção de HP é baixa, podendo aparentar normalidade, diferentemente da TC, a qual apresenta achados anormais em cerca de $90 \%$ dos pacientes com características clínicas. Os achados que podem ser vistos pela TC incluem opacidade em padrão vidro fosco, ou ground glass opacities (GGO), bilateral, simétrico ou irregular na parte média para a parte inferior dos pulmões ou em um padrão broncovascular; múltiplos nódulos centrolobulares frequentemente mal definidos; atenuação em mosaico; e uma combinação de opacidade em padrão vidro fosco irregular, pulmão normal e aprisionamento de ar, também conhecido como "sinal de queijo de cabeça". ${ }^{2}$

Em um estágio mais avançado da HP pode ocorrer dano pulmonar irreversível ou fibrose com reticulação e faveolamento, perda de volume predominante no lobo superior, linfadenopatia mediastinal, aumento da artéria pulmonar, enfisema intermediário do pulmão ou enfisema centrolobular difuso, poupando os ápices e bases extremos, bronquiectasia de tração e, também, bronquiolectasia. $^{2}$

Já tratando-se da infecção pelo SARS-CoV-2, inúmeros estudos apontam um padrão existente entre diversos achados de imagem no COVID-19, que inclui, comumente, opacidades em vidro fosco, consolidativas ou nodulares, com o tipo de distribuição sendo periférico, bilateral e multilobar. Achados adicionais incluem padrão de pavimentação em mosaico, ou crazy paving (linhas interlobulares e intralobulares espessas sobrepostas em um fundo de opacidades com aspecto de vidro fosco), espessamento do septo interlobular, bronquiectasia ou envolvimento subpleural. Assim, estabeleceu-se, segundo a Radiological Society of North America (RSNA), um padrão típico atribuído à pneumonia por COVID-19, o qual é achado de opacidade em padrão vidro fosco em uma distribuição periférica bilateral, com ou sem consolidação ou linhas intralobulares visíveis (padrão pavimentação em mosaico). Assim, nota-se certa semelhança de achados entre os casos de pneumonia por COVID-19 e a pneumonite de hipersensibilidade. ${ }^{2}$

Nessa linha, é importante mencionar que os achados radiológicos e patológicos das reações inflamatórias pulmonares de indivíduos infectados pelo novo Coronavírus, com desenvolvimento de pneumonia, são mais semelhantes ao de pessoas com pneumonite de hipersensibilidade, devido à hipersensibilidade 
pulmonar, do que com outras infecções virais. ${ }^{4}$ Tal fato evidencia ainda mais a relação existente entre HP e a COVID-19.

Figura 2. Caso de pneumonite por hipersensibilidade subaguda. Imagem axial de TC (A) demonstra opacidades em padrão vidro fosco bilaterais multifocais em ambos os pulmões (setas brancas), com áreas de pavimentação em mosaico (seta preta). A imagem coronal (B) mostra os mesmos achados com preservação dos ápices pulmonares. ${ }^{2}$
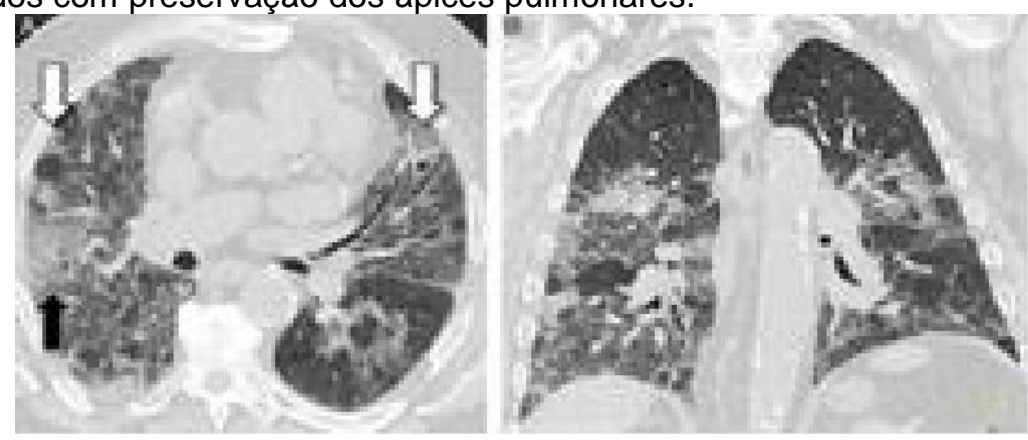

Figura 3. Progressão temporal do COVID-19. Imagens axiais de TC (A, B) realizadas no dia 5 dos sintomas mostram consolidação periférica e peribrônquica multifocal bilateral e opacidade em padrão vidro fosco. Imagens axiais de TC (C, D) realizadas 21 dias depois mostram melhora da consolidação com opacidade em padrão vidro fosco residual e padrão reticular nos lobos superiores, coalescência de consolidação em ambos os lobos inferiores (retângulo) e desenvolvimento de estrias fibróticas lineares (setas) e uma pequena esquerda derrame pleural (retângulo). ${ }^{2}$
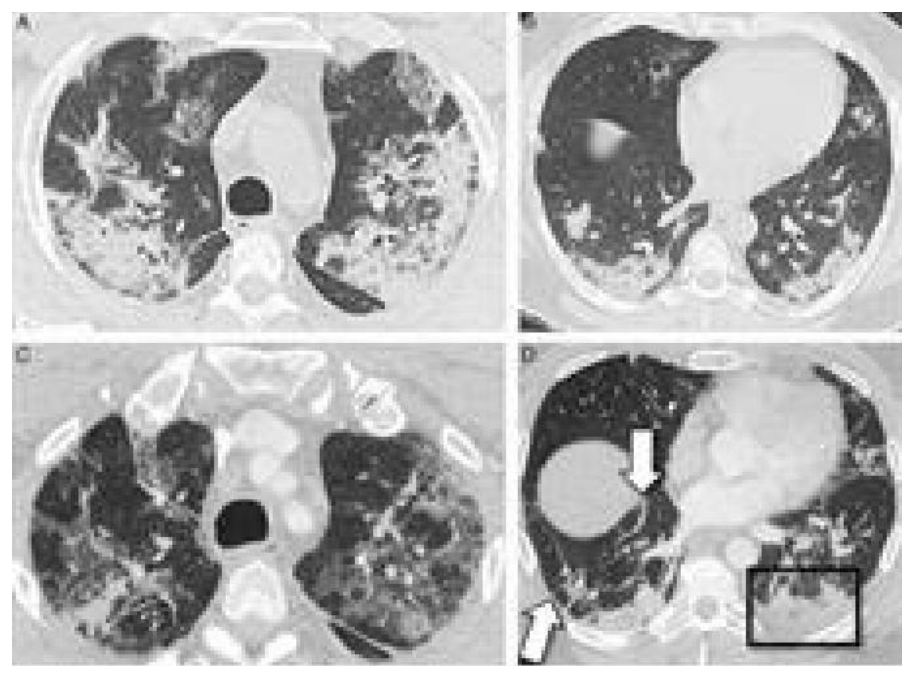

\subsection{TRATAMENTO}

A estratégia tradicional de tratamento para a pneumonite de hipersensibilidade é combater o antígeno, no caso do SARS-Cov-2 por meio da redução da carga viral, e controlar a resposta imune à infecção. Medicamentos antivirais são utilizados como formas de reduzir tanto a disseminação viral quanto o potencial virulento, entre eles os inibidores de nucleotídeos, inibidores da transcriptase reversa análogos e não análogos de nucleosídeo (ITRN e ITRNN) 
do vírus da imunodeficiência humana (HIV), inibidor da protease do HIV e sulfato de dextrana. ${ }^{1}$

Em casos de HP aguda, medicamentos que regulam a cascata do complemento são considerados. Ademais, comprimidos orais de esteróides em baixa dosagem alcançam notoriedade na profilaxia e terapêutica quando usados no estágio inicial da COVID-19. Já com a progressão e gravidade da doença, esteróides em alta dose podem diminuir a fibrose pulmonar e, assim, a mortalidade. ${ }^{1}$

Tratando-se do uso dos corticosteróides, pesquisas preconizam a metilprednisolona (MP) em dose média ou alta, inclusive nos pacientes que necessitam de ventilação mecânica, a fim de evitar a progressão da hipersensibilidade pulmonar e prevenir a insuficiência respiratória hipóxica, provocada pelo COVID-19 na fase grave e realizar o desmame do ventilador. Observou-se que, nesses casos, a aplicação de MP aumentou significativamente a relação entre $\mathrm{PaO} 2 / \mathrm{FiO} 2$, estimulando respostas positivas no combate à insuficiência respiratória. ${ }^{4}$ Ao realizar um tratamento com corticosteróides para 0 COVID-19 deve-se ter cautela, uma vez que o seu uso em baixa dose por um período de tempo maior que 10 dias pode suprimir o estado imunológico natural do organismo, dificultando a remoção do SARS-CoV-2. ${ }^{4}$

Nota-se, por fim, uma individualidade em cada técnica terapêutica, que deve ser adaptada de acordo com a idade do paciente, comorbidades presentes, medicamentos de uso contínuo e estado imunológico.

\section{CONCLUSÃO}

A partir da leitura e análise dos artigos pesquisados, conclui-se que existe um padrão típico radiológico e patológico atribuído à pneumonia do COVID-19 que se assemelha ao que é verificado na pneumonite por hipersensibilidade. Nos achados radiológicos, destacam-se as opacidades em padrão vidro fosco, consolidativas ou nodulares, em uma distribuição periférica bilateral, podendo ser sobrepostas por linhas interlobulares e intralobulares espessas sobrepostas (padrão pavimentação em mosaico), bronquiectasia e fibrose pulmonar. Clinicamente, pode-se observar comprometimento da troca de gases, levando, à Síndrome do Desconforto Respiratório Agudo, e, possivelmente, associando-se à 
insuficiência ventricular direita e ao risco aumentado de tromboembolismo pulmonar.

Diante desse cenário, é válido ressaltar que o vírus SARS-COV-2 recebeu atenção global especialmente por sua alta transmissibilidade e por suas graves manifestações, principalmente entre os indivíduos que se enquadram nos grupos de risco (idosos, indivíduos com doenças crônicas e/ou imunossupressoras, entre outros). Outrossim, apesar do atual contexto pandêmico influenciar nas suspeitas de diagnóstico, principalmente devido às inflamações pulmonares encontradas nos exames radiológicos e patológicos de pacientes acometidos pela COVID-19 e pela hipersensibilidade pulmonar, é necessária e imprescindível a confirmação dos achados por meio da realização de exames específicos.

Desse modo, cada estratégia de tratamento respeitará a individualidade do paciente, incluindo idade, comorbidades presentes, medicamentos de uso contínuo e estado imunológico e, consequentemente, terá maior taxa de sucesso de cura. Portanto, atenção, conhecimento, precisão e cautela auxiliarão no encaminhamento para a regressão da doença. 


\section{REFERÊNCIAS}

Song, young goo et al. Covid-19, a clinical syndrome manifesting as hypersensitivity pneumonitis. Infect chemother, [s. L.], v. 52, n. 1, p. 110-112, 10 mar. 2020. Doi 10.3947/ic.2020.52.1.110. Disponível em: https://pubmed.ncbi.nlm.nih.gov/32153144/. Acesso em: 13 set. 2021

Hanfi, sammer $h$ et al. Covid-19 and its mimics: what the radiologist needs to know. $\mathrm{J}$ thorac imaging, [s. L.], v. 36, n. 1, p. W1-w10, jan. 2021. Doi 10.1097/rti.0000000000000554. Disponível em: https://pubmed.ncbi.nlm.nih.gov/32852419/. Acesso em: 13 set. 2021

Sanchez-gonzalez, marcos a et al. A pathophysiological perspective on covid-19's lethal complication: from viremia to hypersensitivity pneumonitis-like immune dysregulation. Infect chemother, [s. L.], v. 52, n. 3, set. 2020. Doi 10.3947/ic.2020.52.3.335. Disponível em: https://pubmed.ncbi.nlm.nih.gov/32537960/. Acesso em: 13 set. 2021.

Chen, meizhu et al. Hypersensitivity in the lungs is responsible for acute respiratory failure in covid-19 patients: case series of patients who received high-dose/shortterm methylprednisolone. Clin transl allergy, [s. L.], v. 11, n. 7, 27 ago. 2021. Doi 10.1002/clt2.12056. Disponível em: https://pubmed.ncbi.nlm.nih.gov/34484687. Acesso em: 13 set. 2021. 